\title{
Relationship between health-related quality of life, perceived family support and unmet health needs in adult patients with multimorbidity attending primary care in Portugal: a multicentre cross-sectional study
}

Filipe Prazeres ${ }^{1,2^{*}}$ and Luiz Santiago ${ }^{1,3}$

\begin{abstract}
Background: Multimorbidity has a high prevalence in the primary care context and it is frequently associated with worse health-related quality of life (HRQoL). Few studies evaluated the variables that could have a potential effect on HRQoL of primary care patients with multimorbidity. The purpose of this study, the first of its kind ever undertaken in Portugal, is to analyse the relationship between multimorbidity, health-related quality of life, perceived family support and unmet health needs in adult patients attending primary care.

Methods: Multicentre, cross-sectional survey conducted among primary care patients with multimorbidity. It included 521 participants (64.1\% females) who met the inclusion criteria. HRQoL was evaluated using the Portuguese Short Form-12 Health Status Questionnaire. The Portuguese Family APGAR was used to measure the perceived family support. A patients' unmet health needs questionnaire was used. The unmet needs for medical, surgical and dental care; prescription medications; mental healthcare or counselling; and eyeglasses or other technical aid was assessed. Descriptive and multivariate analyses were performed.

Results: The sample had an overall average of 4.5 chronic health problems. Increased multimorbidity levels were linked to worse health-related quality of life, particularly the physical health. Some variables were confirmed as playing a role on health-related quality of life. Male patients with high monthly incomes and highly functional families had better physical and mental health. High levels of education and the presence of asthma were also associated with better physical health. Contrariwise, elderly patients with high levels of multimorbidity and with osteoarthritis had lower physical health. The majority of the patients did not have unmet health needs. When health needs were stated they were mostly for generalist medical care, dental care, and eyeglasses/other technical aid. Financial insufficiency was the primary reason for not fulfilling their health needs.

(Continued on next page)
\end{abstract}

\footnotetext{
* Correspondence: filipeprazeresmd@gmail.com

${ }^{1}$ Faculdade de Ciências da Saúde, Universidade da Beira Interior, Covilhã 6200-506, Portugal

${ }^{2}$ Centro de Saúde de Aveiro, Aveiro 3810-000, Portugal

Full list of author information is available at the end of the article
} 
(Continued from previous page)

Conclusion: To improve the quality of life of multimorbid patients, within primary care practices and health delivery systems, one should take into special account the sex of the patient, the perceived family support and the self-perceived economic status because of their relationship with both physical and mental health. Limitations and recommendations are discussed.

Keywords: Multimorbidity, Health-related quality of life, Family support, Unmet health needs, Primary health care, Portugal

\section{Background}

The prevalence of multimorbidity, defined as the cooccurrence of 2 or more chronic health problems within one person [1], is increasing worldwide due to the effects of improved living conditions, better medical care and an aging population [2, 3]. Portuguese epidemiologic data follows the same tendency, with a high prevalence of multimorbidity (72.7 \%) amongst adult patients attending primary care [4]. Factors such as social deprivation [5], marginalisation [6], mental health disorders [5], and poor housing conditions [7] are associated with an increased prevalence of multimorbidity.

Living with multimorbidity can be a difficult task for the patients as well as for their healthcare providers. Multimorbid patients are more likely to die early [8], experience poor clinical outcomes [9] and a decline of physical functioning [10]. When describing the health burden of chronic diseases, healthcare providers should include measurements of health-related quality of life (HRQoL) [11].

HRQoL is a multidimensional concept that includes domains related to physical, mental, emotional and social functioning associated with an illness or treatment [12]. The Centers for Disease Control and Prevention (CDC) defined HRQoL as an individual's or group's perceived physical and mental health over time [13]. Selfrated health status is also a predictor of mortality [14].

Since multimorbidity has a significant negative impact on HRQoL [15-19], it would be expected that this relationship would be commonly researched, particularly in the primary care context where the majority of treated patients are multimorbid [20], but this is not the case [16]. Limited information exists about the influence of sociodemographic factors (e.g. social support, educational background, economic status) on HRQoL of primary care patients with multimorbidity [2]. There is some evidence to suggest that strong social support from family members can protect against illness or disability [21] and improve chronic illness outcomes [22].

Therefore, a comprehensive approach to the multimorbid patient should take into consideration not only the measurement of HRQoL, but also the impact of the different sociodemographic factors on HRQoL $[2,16]$, including family support, and the health needs of this group of patients, for GPs to improve care to multimorbid patients and ultimately improve the efficacy of healthcare planning and deal with the inherent social costs, particularly in contexts with limited resources [23].

The purpose of the present study, the first of its kind ever undertaken in Portugal, is to determine the impact of multimorbidity on HRQoL in patients aged 18 years and above attending primary care. Specifically, this study aims to i) characterise the unmet health needs of adult patients with multimorbidity, ii) assess family support to adult patients with multimorbidity; iii) analyse the relationship between multimorbidity, patients' health-related quality of life, perceived family support and unmet health needs.

\section{Methods \\ Study design}

A multicentre, cross-sectional survey was conducted among primary care patients with multimorbidity in thirteen Primary Care Centres in the Centre region of Portugal, between January 2014 and January 2015. This study reports on Phase II of a Three-Phase project (MM-PT: Multimorbidity in primary care in PorTugal) aimed at raising awareness on the relevance to deal with multimorbidity in Portugal. Details regarding the full project's protocol were previously published elsewhere [24].

The study was approved by local research ethics committees (Faculty of Health Sciences - University of Beira Interior - and the Central Health Region of Portugal) and was conducted in accordance with the principles of the Declaration of Helsinki [25]. Informed consent was obtained from all participants and confidentiality was maintained.

The reporting of this study conforms to the Strengthening the Reporting of Observational Studies in Epidemiology (STROBE) statement [26].

\section{Sample}

Study size and sampling of the Primary Care Centres have been described elsewhere [24]. Enrolled GPs recruited patients presenting for a primary care appointment at each 
of the institutions during the period of the study. They ensured that each individual patient met the criteria for eligibility. Participation inclusion criteria included being a willing volunteer to participate; being 18 or more years of age; and having a recorded diagnosis of at least two chronic health problems, of which at least one was required to be hypertension, diabetes, asthma or osteoarthritis. These 4 diseases were selected because, on the one hand, they have high prevalence and are frequently associated with low quality of life, and on the other hand, there are national efforts to implement specific recommendations for the diagnosis, treatment and control of these diseases [24]. Exclusion criteria included being acutely unwell or presenting inability to provide independent informed consent. A total of 548 patients were approached (including approximately $10 \%$ above estimated sample size to account for expected missing data). Twenty-seven individuals refused to participate without any stated reason. Therefore, 521 interviews were performed; all interviews were fully completed and so no missing data was encountered.

\section{Data collection procedures}

Data collection was performed by protocol [24]. It was carried out through a structured face-to-face interview delivered by the investigator or a GP/GP trainee. In order to minimize interview bias, all interviewers were very experienced in conducting face-to-face interviews and, if needed, received additional training from members of the research team. Interviews were performed after the patient's clinical visit or while waiting for their appointment. Consenting patients were evaluated at a single-time point and the responses were recorded on paper. The average time of the interview was $15 \mathrm{~min}$.

\section{Measures}

\section{Sociodemographic characteristics}

Using the personal information section of the Portuguese version of the EASY-Care questionnaire [27], self-reported data were obtained for sex (male/female), age group (18-34/35-49/50-64/ $\geq 65$ years), residence area (urban/ rural), current marital status (married-cohabiting/single/widowed/separated-divorced), number of years of formal education (less than 6 years/at least 6 but not more than 9 years/more than 9 years), living arrangements (couple/extended family/alone/other), professional status (pensioner-retired/employed/unemployed/ housewife/student) and self-perceived economic status ("Just enough to make ends meet"/"Not enough to make ends meet"/"Some money left over").

\section{Medical history and measures of multimorbidity}

Patients' chronic health problems were collected by the investigator or a GP/GP trainee using 3 data sources for each patient: GPs knowledge of patient's history, patient's self-report and medical records.

The current study considered the 147 International Classification of Primary Care (ICPC-2) diagnoses gathered by O'Halloran et al. [28] (Family Medicine Research Centre, University of Sydney). These diagnoses were defined as chronic by the O'Halloran criteria: a) have a duration that has lasted, or is expected to last, at least 6 months; b) have a pattern of recurrence, or deterioration; c) have a poor prognosis and d) produce consequences, or sequelae that impact on the individual's quality of life [28].

Multimorbidity was measured based on simple counts of chronic health problems coexisting within one person. Drawing on the categorization of Kadam et al. [29], multimorbidity was classified into low morbidity count (2 or 3 chronic health problems), medium (4 or 5 chronic health problems) and high (6 or more chronic health problems). No assessment of disease severity in the multimorbid conditions found was undertaken.

\section{Health-related quality of life}

The Portuguese Short Form-12 Health Status Questionnaire (SF-12) [30], was used to assess health-related quality of life from the patient's perspective.

The SF-12 [31] is a short form survey with 12 questions. In studies with large samples $(n=500)$ it is a valid alternative to the 36-item Short Form (SF-36) [31] since it takes an average of $2 \mathrm{~min}$ to administer and has a reduced respondent and administrative burdens [31-33]. The SF-12 addresses the same 8 domains as identified in the SF-36: physical functioning (PF, 2 items); role limitations due to physical health problems (RP, 2 items); bodily pain (BP, 1 item); general health perceptions $(\mathrm{GH}, 1$ item); vitality (VT, 1 item); social functioning (SF, 1 item); role limitations due to emotional problems (RE, 2 items) and mental health (MH, 2 items). The SF-12 also assesses 2 health status composite scores: physical health (Physical Component Summary, PCS) and mental health (Mental Component Summary, MCS). These composite scores are generated using an algorithm for comparison to normative data-general United States (US) population-with a mean score of 50 and a standard deviation of 10; scores above 50 indicate better physical or mental health and scores bellow 50 indicate worse health [31]. Since there is little difference between standard scoring algorithms (US-derived) and country-specific algorithms, the use of the standard scoring algorithms is recommended to allow data comparisons across countries [34].

This brief tool (SF-12) has been used extensively in clinical and population-based studies [32], including those with chronic health conditions. The Portuguese version has shown satisfactory reliability and validity [30]. In the present study, both summary measures 
exceeded the 0.70 level for Cronbach's Alpha (internal consistency) indicating satisfactory results ( $\alpha$ for the PCS and the MCS was 0.84 and 0.82 , respectively).

\section{Family support}

The Portuguese Family APGAR Questionnaire [35, 36] was used to measure the perceived family support of patients with multimorbidity. This questionnaire is commonly used in the Portuguese primary care setting, since it is widely available to GPs as an integral part of the electronic health records software.

Family APGAR Questionnaire features five closedended questions measuring family member's satisfaction with each of the five basic components of family function (Adaptation, Partnership, Growth, Affection and Resolve) [37]. The response format is a three-point scale ("almost always"-two points; "some of the time"-one point; or "hardly ever"-zero points). The scores for each of the five questions after totaled originate the following categories: a) severely dysfunctional families (0 to 3 points); b) moderately dysfunctional families (4 to 7 points); or c) highly functional families (8 to 10 points) $[37,38]$. In the study, Cronbach's Alpha (internal consistency) for the total scale was 0.86 .

\section{Patients' unmet health needs}

The unmet needs for medical, surgical and dental care; prescription medications; mental healthcare or counselling; and eyeglasses or other technical aid were evaluated. The detailed set of questions used in this study are provided in the previously published protocol [24]. These questions were pilot tested for comprehensibility in 50 adult general practice patients, no changes were necessary.

\section{Statistical analyses}

Variables were summarized using descriptive statistics namely absolute (n) and relative (\%) frequencies for categorical variables and mean and standard deviation (SD) for numerical variables.

Univariate analyses were performed to study the association between presence of unmet health needs, presence of moderate/severe dysfunctional family and health related quality of life with patients' characteristics using Chi-square test (categorical variables) or Kruskal-Wallis test (numerical variables which did not follow normal distribution).

Multiple binary logistic regression for presence of unmet health needs and perceived moderately/severely dysfunctional family was performed using variables found to be statistically significant in the univariate analysis and a stepwise selection method (variables were entered considering a stepwise probability of 0.05 ). Pairwise comparisons within comorbidity groups were performed using Dunn's [39] procedure with a Bonferroni correction for multiple comparisons.

Multiple linear regression was performed for PCS and MCS scores using variables significant in the univariate analysis and a stepwise selection method (variables were entered considering a stepwise probability of 0.05 ).

All tests were two-sided considering a significance level of 0.05. Statistical analysis was performed using IBM SPSS Statistics for Windows, Version 21.0 (IBM Corporation, Armonk, NY, USA).

\section{Results}

\section{Characteristics of participants}

Demographic and medical characteristics of the 521 study participants are shown in Table 1 . Mean age was 58.2 years (61.2 years for men and 56.6 years for women). The majority of participants were female $(64.1 \%)$ and $57.2 \%$ had a low level of education. Approximately half of those surveyed (46.3\%) reported a sufficient monthly income. Seventy per cent were married or cohabiting, and $54.3 \%$ lived as a couple.

Low morbidity count was present in $42.2 \%$ of the sample, $27.6 \%$ had a medium morbidity count and a high morbidity count was encountered in $30.1 \%$, with an overall average of 4.5 chronic health problems per participant (4.6 in men and 4.5 in women).

\section{Unmet health needs and multimorbidity}

Unmet health needs are described in Table 2. At least one unmet health need in the preceding 12 months was reported by about one third of the patients and $7.3 \%$ reported two or more unmet health needs.

The most common unmet health needs were related to generalist medical care, dental care, and eyeglasses or other technical aid. The most frequently cited reason for explaining the presence of unmet health needs was financial (18\% of the respondents had to spend their money for food, clothing, housing, etc.).

Presence of unmet health needs was statistically similar across the three multimorbidity groups $(p=0.676)$ (Table 2).

\section{Unmet health needs and other characteristics}

From univariate analysis, presence of unmet needs was more frequently reported by women than men $(37.7 \%$ vs. $18.7 \% ; p<0.001$ ), by patients with lower/medium education levels than higher level (33.3\% vs. $23.0 \%$; $p=0.030$ ), by patients with insufficient monthly income than by sufficient/higher monthly incomes ( $48.2 \%$ vs. $22.4 \% / 13.6 \% ; p<0.001$ ), by non-diabetics than diabetic patients (33.8 \% vs. $23.8 \% ; p=0.026)$, and by patients with osteoarthritis than without it ( $35.7 \%$ vs. $24.4 \% ; p=0.006$ ). Moreover, patients reporting unmet health needs were 5 years younger than patients 
Table 1 Demographic and medical characteristics of participants $(n=521)$

\begin{tabular}{|c|c|}
\hline \multicolumn{2}{|l|}{ Sex, \% (n) } \\
\hline Women & $64.1(334)$ \\
\hline Men & $35.9(187)$ \\
\hline \multicolumn{2}{|l|}{ Age group, \% (n) } \\
\hline 18-34 years & $9.0(47)$ \\
\hline $35-49$ years & $15.7(82)$ \\
\hline $50-64$ years & $39.5(206)$ \\
\hline$\geq 65$ years & $35.7(186)$ \\
\hline \multicolumn{2}{|l|}{ Residence area, \% (n) } \\
\hline Urban & $49.1(256)$ \\
\hline Rural & $50.9(265)$ \\
\hline \multicolumn{2}{|l|}{ Marital status, \% (n) } \\
\hline Married/cohabiting & $70.2(366)$ \\
\hline Single & $11.5(60)$ \\
\hline Widowed & $8.6(45)$ \\
\hline Separated/divorced & $9.6(50)$ \\
\hline \multicolumn{2}{|l|}{ Living arrangements, \% ( $n)$} \\
\hline Couple & $54.3(283)$ \\
\hline Extended Family & $31.3(163)$ \\
\hline Alone & $11.9(62)$ \\
\hline Other (including care home) & $2.5(13)$ \\
\hline \multicolumn{2}{|l|}{ Education, \% ( $n)$} \\
\hline Low level (less than 6 years) & $57.2(298)$ \\
\hline Medium level (at least 6 but not more than 9 years) & $19.4(101)$ \\
\hline High level (more than 9 years) & $23.4(122)$ \\
\hline \multicolumn{2}{|l|}{ Professional status, \% ( $n)$} \\
\hline Pensioner/retired & $43.0(224)$ \\
\hline Employed (full-time/part time) & $34.2(178)$ \\
\hline Unemployed & $11.3(59)$ \\
\hline Housewife & $10.4(54)$ \\
\hline Student & $1.2(6)$ \\
\hline \multicolumn{2}{|l|}{ Monthly income, \% (n) } \\
\hline "Not enough to make ends meet" & $38.2(199)$ \\
\hline "Just enough to make ends meet" & $46.3(241)$ \\
\hline "Some money left over" & $15.5(81)$ \\
\hline \multicolumn{2}{|l|}{ Multimorbidity group, \% (n) } \\
\hline Low (2-3 chronic problems) & $42.2(220)$ \\
\hline Medium (4-5 chronic problems) & $27.6(144)$ \\
\hline High ( $\geq 6$ chronic problems) & $30.1(157)$ \\
\hline \multicolumn{2}{|l|}{ Chronic health problems ${ }^{\mathrm{a}}, \%(n)$} \\
\hline Hypertension & $61.8(322)$ \\
\hline Diabetes mellitus & $29.0(151)$ \\
\hline Asthma & $17.3(90)$ \\
\hline Osteoarthritis & $57.6(300)$ \\
\hline
\end{tabular}

Table 1 Demographic and medical characteristics of participants $(n=521)$ (Continued)

Other prevalent chronic health problems ${ }^{\mathrm{a}}, \%(n)$

\begin{tabular}{ll} 
Lipid disorder & $63.1(329)$ \\
Depressive disorder & $19.6(102)$ \\
Obesity & $14.2(74)$ \\
Overweight & $10.9(57)$ \\
Varicose veins of leg & $9.8(51)$ \\
Benign prostatic hypertrophy & $8.1(42)$ \\
Osteoporosis & $7.9(41)$ \\
Goitre & $7.7(40)$ \\
Liver disease & $7.1(37)$ \\
Anxiety disorder/anxiety state & $5.4(28)$ \\
\hline
\end{tabular}

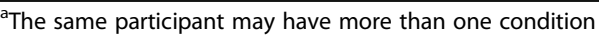

without unmet needs (average/range: 55 years/20-92 years vs. 60 years $/ 18-93$ years; $p=0.003$ ).

Multivariate analysis (Table 3) shows that variables remaining important in explaining the presence of unmet health needs were sex, age, monthly income and education level. Women were 2.3 times more likely to report unmet health needs than men. Patients aged 1834 years were 2.5 times more likely to report unmet health needs than older patients. Patients with insufficient monthly income were nearly 3.3 times more likely to report unmet health needs. Patients with low/medium level of education were 2 times more likely to report unmet health needs. The presence of diabetes or osteoarthritis was not statistically significant to the model.

\section{Perceived family support and multimorbidity}

Regarding the family support as reported by the sample, the majority $(70.4 \%)$ perceived their families to be highly functional, $20.3 \%$ reported as being moderately dysfunctional and $9.2 \%$ severely dysfunctional (Table 2). On a scale of 0 to 10 (where 0 corresponds to the lowest and 10 to the highest family support) this represents a mean (SD) of 7.9 (2.7) for the overall sample.

According to the multimorbidity range, the group of patients with a high morbidity count (6 or more chronic health problems) had slightly higher perception of having a dysfunctional family than the low and medium multimorbidity groups; although this difference was not statistically significant $(p=0.363)$ (Table 2 ).

\section{Perceived family support and other characteristics}

From univariate analysis, perception of family dysfunction (moderate/severe) was more frequently reported by women than men ( $35.6 \%$ vs. $18.7 \%$; $p<0.001)$, by patients living in urban that rural areas $(33.6 \%$ vs. $25.7 \%$; $p=0.047)$, by not married than married patients $(45.2 \%$ vs. $23.0 \%$; $p<0.001)$, by patients with insufficient 
Table 2 Unmet health needs, perceived family support and health related quality of life

\begin{tabular}{|c|c|c|c|c|c|}
\hline & \multirow{2}{*}{$\begin{array}{l}\text { Overall } \\
{[n=521]}\end{array}$} & \multicolumn{3}{|c|}{ Multimorbidity Group } & \multirow[b]{2}{*}{$P$-value } \\
\hline & & $\begin{array}{l}\text { Low } \\
{[n=220]}\end{array}$ & $\begin{array}{l}\text { Medium } \\
{[n=144]}\end{array}$ & $\begin{array}{l}\text { High } \\
{[n=157]}\end{array}$ & \\
\hline \multicolumn{6}{|c|}{ No. of unmet needs by participant, \% (n) } \\
\hline 0 & $69.1(360)$ & $69.1(152)$ & $66.7(96)$ & $71.3(112)$ & 0.676 \\
\hline 1 & $23.6(123)$ & $23.2(51)$ & $25.7(37)$ & $22.3(35)$ & \\
\hline 2 & $6.0(31)$ & $6.8(15)$ & $5.6(8)$ & $5.1(8)$ & \\
\hline 3 & $0.8(4)$ & $0.5(1)$ & $0.7(1)$ & $1.3(2)$ & \\
\hline 4 & $0.6(3)$ & $0.5(1)$ & $1.4(2)$ & $0.0(0)$ & \\
\hline \multicolumn{6}{|l|}{ Type of unmet needs, $\%(n)^{\mathrm{a}}$} \\
\hline Prescription medications & $1.2(6)$ & $0.9(2)$ & $2.8(4)$ & $0.0(0)$ & n.a. \\
\hline General medical care & $13.1(68)$ & $13.2(29)$ & $16.0(23)$ & $10.2(16)$ & 0.330 \\
\hline Surgical care & $1.0(5)$ & $0.5(1)$ & $1.4(2)$ & $1.3(2)$ & n.a. \\
\hline Mental healthcare/counselling & $1.0(5)$ & $0.0(0)$ & $2.8(4)$ & $0.6(1)$ & n.a. \\
\hline Dental care & $12.7(66)$ & $13.2(29)$ & $11.1(16)$ & $13.4(21)$ & 0.803 \\
\hline Eyeglasses/technical aid & $11.3(59)$ & $12.3(27)$ & $10.4(15)$ & $10.8(17)$ & 0.838 \\
\hline \multicolumn{6}{|l|}{ Reasons, \% (n) ${ }^{\mathrm{a}}$} \\
\hline Professional & $3.1(16)$ & $5.5(12)$ & $2.8(4)$ & $0.0(0)$ & n.a. \\
\hline Too sick & $1.2(6)$ & $1.4(3)$ & $1.4(2)$ & $0.6(1)$ & n.a. \\
\hline Mobility & $1.7(9)$ & $0.5(1)$ & $2.8(4)$ & $2.5(4)$ & n.a. \\
\hline Care provider of a dependent & $1.2(6)$ & $1.4(3)$ & $0.7(1)$ & $1.3(2)$ & n.a. \\
\hline Afraid to leave home & $1.0(5)$ & $0.5(1)$ & $2.8(4)$ & $0.0(0)$ & n.a. \\
\hline Other concerns & $1.0(5)$ & $1.8(4)$ & $0.7(1)$ & $0.0(0)$ & n.a. \\
\hline Financial & $18.0(94)$ & $18.2(40)$ & $16.7(24)$ & $19.1(30)$ & 0.857 \\
\hline Access to GP consultations & $8.6(45)$ & $6.4(14)$ & $12.5(18)$ & $8.3(13)$ & 0.123 \\
\hline \multicolumn{6}{|l|}{ Family APGAR categories, \% ( $n$ ) } \\
\hline Severely Dysfunctional & $9.2(48)$ & $7.3(16)$ & $8.3(12)$ & $12.7(20)$ & 0.363 \\
\hline Moderately Dysfunctional & $20.3(106)$ & $22.3(49)$ & $18.1(26)$ & $19.7(31)$ & \\
\hline Highly Functional & $70.4(367)$ & 70.5 (155) & $73.6(106)$ & $67.5(106)$ & \\
\hline \multicolumn{6}{|l|}{ SF-12 scores, mean (SD) } \\
\hline PF & $44.2(12.4)$ & $48.5(10.8)$ & $42.4(12.4)$ & $39.7(12.6)$ & $<0.001$ \\
\hline $\mathrm{RP}$ & $43.1(12.9)$ & $47.2(11.1)$ & $42.1(13.0)$ & $38.1(13.2)$ & $<0.001$ \\
\hline $\mathrm{BP}$ & $40.0(12.4)$ & $43.6(12.0)$ & $39.1(12.6)$ & $36.0(11.5)$ & $<0.001$ \\
\hline $\mathrm{GH}$ & $34.6(11.2)$ & $39.0(11.5)$ & $32.5(9.9)$ & $30.4(9.6)$ & $<0.001$ \\
\hline VT & $48.0(11.4)$ & $50.3(10.8)$ & $47.1(11.3)$ & $45.7(11.6)$ & $<0.001$ \\
\hline SF & $44.8(13.5)$ & $47.5(12.7)$ & $43.8(13.5)$ & $41.8(14.1)$ & $<0.001$ \\
\hline RE & $44.1(12.6)$ & $46.3(11.3)$ & $44.2(12.8)$ & $40.8(13.5)$ & $<0.001$ \\
\hline $\mathrm{MH}$ & $45.1(12.9)$ & $46.9(12.5)$ & $45.5(12.7)$ & $42.3(13.1)$ & 0.002 \\
\hline PCS & $40.3(11.9)$ & $45.0(11.0)$ & $38.3(11.3)$ & $35.5(11.1)$ & $<0.001$ \\
\hline MCS & $46.6(12.2)$ & $47.8(12.0)$ & $47.0(12.3)$ & $44.6(12.4)$ & 0.033 \\
\hline
\end{tabular}

n.a. - Chi-square test not applicable due to low frequencies

$P F$ physical functioning, $R P$ role physical, $B P$ bodily pain, $G H$ general health, $V T$ vitality, $S F$ social functioning, $R E$ role emotional, $M H$ mental health, $P C S$ physical component summary, MCS mental component summary

${ }^{a}$ The same participant may have reported more than one option 
Table 3 Multiple logistic regression for presence of unmet health needs

\begin{tabular}{lll}
\hline Factors & OR $(95 \% \mathrm{Cl})$ & -Value \\
\hline $\begin{array}{l}\text { Sex } \\
\quad \text { Women }\end{array}$ & $2.33(1.48-3.66)$ & $<0.001$ \\
$\begin{array}{l}\text { Age group } \\
\quad \text { 18-34 years }\end{array}$ & $2.47(1.21-5.05)$ & 0.013 \\
$\begin{array}{l}\text { Monthly income } \\
\quad \text { Not enough to make ends meet" }\end{array}$ & $3.29(2.17-4.99)$ & $<0.001$ \\
$\begin{array}{l}\text { Education } \\
\text { Low/medium level }\end{array}$ & $2.03(1.15-3.58)$ & 0.015
\end{tabular}

Reference category: sex = male; monthly income = "Just enough to make ends meet" aggregated with "Some money left over"; age group = higher than 34 years; education $=$ high level

monthly income than with sufficient/higher monthly incomes $(40.7 \%$ vs. $25.3 \% / 14.8 \% ; p<0.001)$, by patients living alone than with an extended family (54.8\% vs. $22.7 \%, p<0.001)$ and by patients with unmet health needs than without them (44.1\% vs. $23.1 \%$; $p<0.001)$.

Table 4 shows that variables remaining associated with perception of family dysfunction in multivariate analysis were sex, marital status, monthly income, living arrangements and presence of unmet needs. Women were 2 times more likely to perceive a dysfunctional family than men. Single/divorced/widow patients were 2.8 times more likely to perceive a dysfunctional family than married patients. Patients with insufficient monthly income were 1.8 times more likely to perceive a dysfunctional family. Patients with unmet needs were 1.9 times more likely to report a dysfunctional family. Patients living alone are more likely to perceive a dysfunctional family than patients living in an extended family.

Table 4 Multiple logistic regression for perceived moderately/ severely dysfunctional family

\begin{tabular}{llc}
\hline Factors & OR $(95 \% \mathrm{Cl})$ & $P$-Value \\
\hline $\begin{array}{l}\text { Sex } \\
\quad \text { Women }\end{array}$ & $2.01(1.26-3.20)$ & 0.003 \\
$\begin{array}{l}\text { Marital status } \\
\quad \text { Single/divorced/widow }\end{array}$ & $2.77(1.48-5.17)$ & 0.001 \\
$\begin{array}{l}\text { Monthly income } \\
\quad \text { Not enough to make ends meet" }\end{array}$ & $1.81(1.18-2.78)$ & 0.007 \\
Living arrangements & $0.905(0.39-2.09)$ & 0.815 \\
$\quad$ Couple & $0.389(0.19-0.79)$ & 0.009 \\
$\quad$ Extended family & $0.910(0.26-3.22)$ & 0.884 \\
$\quad$ Other & & \\
Unmet needs & $1.94(1.24-3.0)$ & 0.003 \\
\hline
\end{tabular}

Reference category: sex = male; marital status = married; living arrangements = alone; monthly income = "Just enough to make ends meet" aggregated with "Some money left over"; unmet needs = absent
Health-related quality of life and multimorbidity

The majority of the sample had a score bellow 50 (mean of the reference population) in all eight domains of the SF-12, particularly in general health $(91.0 \%)$ and bodily pain (77.9 \%) (Fig. 1). Regarding the two health status composite scores, physical health (PCS) was worse than the mental health (MCS) (Table 2).

All SF-12 scores were statistically related with the multimorbidity groups, showing that health related quality of life decreases when levels of multimorbidity rise (Table 2). Pairwise comparisons revealed no statistically differences between (a) medium versus high morbidity groups in $\mathrm{PF}$ $(p=0.196), \mathrm{BP}(p=0.063), \mathrm{GH}(p=0.226), \mathrm{VT}(p=0.928)$, SF $(p=0.799)$ and PCS scores $(p=0.139)$; (b) medium versus low/high in RE, MH and MCS scores.

\section{Health-related quality of life and other characteristics}

Univariate analysis shows that the SF-12 eight domains and the two health status composite scores were related to at least one participant characteristic besides the multimorbidity level (Table 5). Indeed, all SF-12 scores were statistically related with marital status, monthly income, perceived family support (family APGAR) and the presence of unmet needs (Table 5).

Multivariate analysis for PCS scores (Table 6) shows that sex, age, monthly income, education, multimorbidity groups, family APGAR, osteoarthritis and asthma were statistically significant predictors for PCS score. Male, higher monthly income, higher level of family functionality, higher level of education, younger age, lower levels of multimorbidity, absence of osteoarthritis, and presence of asthma are related with a predicted higher score at PCS. Regarding MCS scores, sex, monthly income, and family APGAR are statistically significant predictors. Being male, having a higher monthly income and a higher level of family functionality are MCS protective factors.

\section{Discussion}

The current study represents the first analysis on healthrelated quality of life among adult patients with multimorbidity in a primary-care context in Portugal.

Globally, the multimorbid sample in this study reported poorer health-related quality of life than the reference population (recommended for international comparisons) [34], which demonstrates the adverse effect of multimorbidity on health-related quality of life. This overall finding is in line with the available literature [15-19, 40]. However, existing studies lack comparable samples and methodologies and no direct comparisons can be made [2].

Health-related quality of life decreased inversely with the number of concurrent chronic health problems, which reflects previous studies [16, 18, 41, 42]. This 


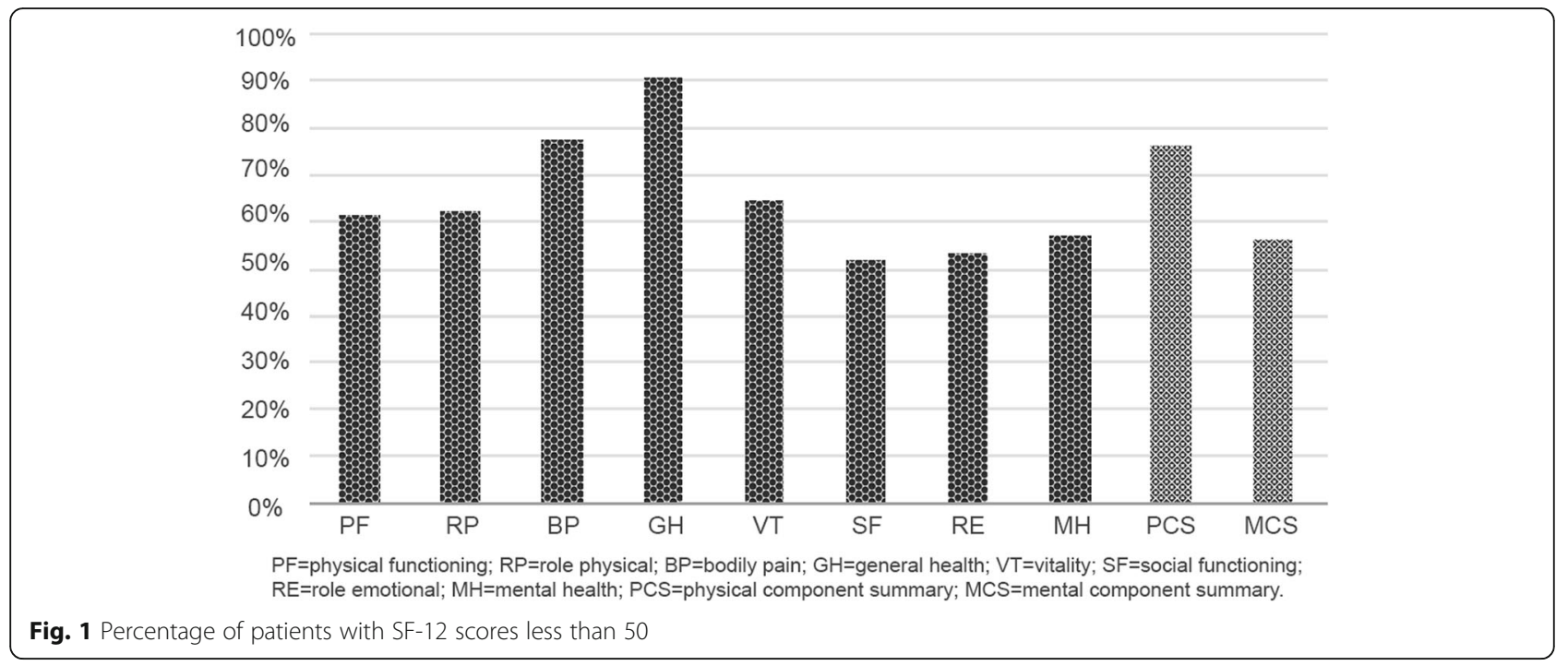

occurrence was particularly evident when comparing the low ( 2 or 3 chronic health problems) and high (6 or more chronic health problems) morbidity count groups for all SF-12 dimensions (the eight domains and the two health status composite scores). Nonetheless, there was only a moderate evidence of the effect of multimorbidity on mental health compared to its effect on physical health, which also seems to be consistent with previous research $[16,17]$.

As could be expected from previous studies [43-45], increasing age was associated with poorer physical health. However, no effect of aging was observed on mental health. This discrepancy can be attributed to the psychological adaptation to illness, over time [46].

Female sex [2, 42, 43, 47, 48], low level of education $[2,43,49]$, and a low income $[15,49,50]$ are commonly associated with impaired health-related quality of life, and the current study's findings corroborate this. An implication of this is the possibility that multimorbid patients may benefit from financial aid through social policy programs.

This study also considered other variables that were earlier pointed out as having a possible impact on health-related quality of life [16] such as marital status,

Table 5 Association between SF-12 scores and participants' characteristics

\begin{tabular}{|c|c|c|c|c|c|c|c|c|c|c|}
\hline \multirow[t]{2}{*}{ Characteristic } & \multicolumn{8}{|c|}{ SF-12 scores $(P$-values* $)$} & \multirow[b]{2}{*}{ PCS } & \multirow[b]{2}{*}{ MCS } \\
\hline & $\mathrm{PF}$ & $\mathrm{RP}$ & $\mathrm{BP}$ & $\mathrm{GH}$ & VT & SF & $\mathrm{RE}$ & $\mathrm{MH}$ & & \\
\hline$\overline{S e x}$ & $<0.001$ & 0.023 & $<0.001$ & 0.016 & 0.004 & $<0.001$ & n.s & $<0.001$ & 0.001 & $<0.001$ \\
\hline Age group & $<0.001$ & $<0.001$ & 0.001 & $<0.001$ & n.s & n.s & n.s & n.s & $<0.001$ & n.s. \\
\hline Living arrangements & 0.010 & 0.009 & n.s & n.s & n.s & 0.025 & n.s & 0.012 & 0.007 & 0.043 \\
\hline Education & $<0.001$ & $<0.001$ & $<0.001$ & $<0.001$ & $<0.001$ & 0.019 & n.s & 0.017 & $<0.001$ & n.s. \\
\hline Residence area & n.s. & n.s & n.s & 0.027 & n.s & 0.045 & n.s & n.s & n.s. & n.s. \\
\hline Marital status & $<0.001$ & 0.001 & 0.001 & $<0.001$ & 0.017 & $<0.001$ & $<0.001$ & 0.003 & $<0.001$ & 0.001 \\
\hline Monthly income & $<0.001$ & $<0.001$ & $<0.001$ & $<0.001$ & $<0.001$ & $<0.001$ & $<0.001$ & $<0.001$ & $<0.001$ & $<0.001$ \\
\hline Professional status & $<0.001$ & $<0.001$ & $<0.001$ & $<0.001$ & 0.021 & n.s & 0.012 & 0.011 & $<0.001$ & n.s. \\
\hline Family APGAR & $<0.001$ & $<0.001$ & $<0.001$ & $<0.001$ & $<0.001$ & $<0.001$ & $<0.001$ & $<0.001$ & $<0.001$ & $<0.001$ \\
\hline Unmet health needs & $<0.001$ & 0.003 & $<0.001$ & $<0.001$ & 0.002 & $<0.001$ & $<0.001$ & $<0.001$ & 0.001 & $<0.001$ \\
\hline Asthma & $<0.001$ & $<0.001$ & $<0.001$ & $<0.001$ & n.s & n.s & n.s & n.s & $<0.001$ & n.s. \\
\hline Osteoarthritis & $<0.001$ & $<0.001$ & $<0.001$ & $<0.001$ & 0.002 & n.s & n.s & 0.023 & $<0.001$ & n.s. \\
\hline Diabetes & 0.002 & 0.047 & n.s & $<0.001$ & n.s & n.s & n.s & n.s & 0.001 & n.s. \\
\hline Hypertension & $<0.001$ & 0.001 & 0.029 & $<0.001$ & n.s & n.s & n.s & n.s & $<0.001$ & n.s. \\
\hline
\end{tabular}

$P F$ physical functioning, $R P$ role physical, $B P$ bodily pain, $G H$ general health, $V T$ vitality, $S F$ social functioning, $R E$ role emotional, $M H$ mental health, $P C S$ physical component summary, MCS mental component summary

* $P$-values are for score comparison between categories of each characteristic (bivariate analysis) 
Table 6 Multiple linear regression for PCS and MCS

\begin{tabular}{|c|c|c|c|c|c|c|}
\hline \multirow[b]{2}{*}{ Variable } & \multicolumn{3}{|l|}{ PCS } & \multicolumn{3}{|l|}{ MCS } \\
\hline & $B$ & $S E_{B}$ & $\beta$ & $B$ & $S E_{B}$ & $\beta$ \\
\hline Intercept & 34.82 & 3.49 & - & 22.02 & 2.41 & - \\
\hline Sex & 3.23 & 0.97 & $0.13^{*}$ & 2.44 & 1.03 & $0.10^{*}$ \\
\hline Age group & -1.73 & 0.65 & $-0.14^{*}$ & - & - & - \\
\hline Monthly Income & 1.71 & 0.67 & $0.10^{*}$ & 2.92 & 0.71 & $0.17^{*}$ \\
\hline Education & 2.28 & 0.68 & $0.16^{*}$ & - & - & - \\
\hline Multimorbidity group & -2.41 & 0.60 & $-0.17^{*}$ & - & - & - \\
\hline Family APGAR & 1.93 & 0.71 & $0.11^{*}$ & 6.16 & 0.78 & $0.33^{*}$ \\
\hline Osteoarthritis & -2.76 & 0.98 & $-0.12^{*}$ & - & - & - \\
\hline Asthma & 3.23 & 1.34 & $0.10^{*}$ & - & - & - \\
\hline
\end{tabular}

$S E_{B}$ standard error of the coefficient, $\beta=$ standardized coefficient

${ }^{*} p<0.05 ; B=$ unstandardized regression coefficient

living arrangements and professional status. The current results do not show a clear relationship between these variables and health-related quality of life. Therefore, further work is still required to clarify the full impact of sociodemographic data on health-related quality of life in patients with multimorbidity [16].

Prior studies have noted the relationship between family APGAR scores and the presence of chronic illness [51, 52]. Despite its multiple chronic health problems, the study's sample reported high family support. Family dysfunction was present at a quite lower proportion than in previous reports [52-54]. This inconsistency may be due to the fact that in previous studies the age of the sample was limited to the geriatric population whereas in this study the age group was $18+$ years old. The established distribution of family support was the same between age groups $(p=0.182)$.

In this study, as expected by previous findings $[55,56]$, perceived family support had an impact on healthrelated quality of life. Multimorbid patients from dysfunctional families reported worse physical and mental health. From these results, it is possible to infer that adult patients with multimorbidity in a primary-care context may have a potential gain in health-related quality of life if family members provide support for their care. In Portugal, most of the support comes from families, more than three quarters of informal caregivers provide daily care [57]. Increased social support from family members improves chronic illness outcomes [22] (e.g. better glycaemic control for diabetic patients [58], better blood pressure control for hypertensive patients [59], and lower disease activity for patients with arthritis [60]). As such, GPs should devise efforts to inform and engage patients' families as partners in the care of the multimorbid patient, notably the women living alone and with an insufficient monthly income.

In the present study, patients with unmet health needs had a statistically significant higher perception of having a dysfunctional family than those without unmet needs. The presence of unmet needs was also associated with lower health-related quality of life. Hence, family intervention programs for multimorbid patients (especially young women with an insufficient monthly income, living alone, and with low/medium level of education) will have to address their needs as to have a significant impact on quality of life and health outcomes [61].

Contrary to expectations, by taking into consideration the sample's morbidity levels, the majority of the patients did not have unmet health needs. But when health needs were stated they were mostly for generalist medical care, dental care, and eyeglasses or other technical aid. Financial insufficiency was the primary reason for not fulfilling their health needs. These findings not only reinforce the previously stated necessity of financial support to multimorbid patients (in particular women), but also that primary care teams should organize resources and schedules to meet the medical care needs of multimorbid patients. Interestingly, younger patients reported greater unmet health needs than older patients. A possible explanation for this finding is the relationship of multimorbidity with higher out-of-pocket spending [62]. Portugal is among the four Organization for Economic Co-operation and Development (OECD) countries with the highest out-of-pocket spending, mostly due to the recently imposed restrictions on tax-deductible expenses [63]. This increase in expenditures affects younger taxpayers and leaves out the older poor patients with tax exemptions. A note of caution is due here, since patients' needs may change as a result of the phase of illness, during major events, periods of disease exacerbation and patient's socioeconomic status. Future studies with a longitudinal approach are therefore recommended.

Several limitations need to be acknowledged. Four chronic health problems have been selected based on their importance and although this excluded patients with multimorbidity without at least one of the selected conditions, the study's sample captured 109 out of a total of 147 possible chronic health problems, a much higher number than the majority of the previously published health-related quality of life studies [16]. The current study did not take into account the severity of each chronic health problem and it had a cross-sectional design, so it was not possible to establish causal relationships. A sample selection bias due to the possibility of non-consecutive recruitment of patients by the GPs, should also be considered.

\section{Conclusion}

The findings of this study link the increased multimorbidity levels to worse health-related quality of life, particularly the physical health, in multimorbid patients aged 18 and older attending primary care consultations. 
Some variables were confirmed as playing a role on health-related quality of life. As a result, to improve the quality of life of multimorbid patients, within primary care practices and health delivery systems, one should take into special account the sex of the patient, the perceived family support and the self-perceived economic status because of their relationship with both physical and mental health. This will also be of relevance when planning longitudinal and interventional studies regarding health-related quality of life.

Further research is suggested on larger nationwide samples to corroborate the results of the current study. It is also recommended to include the quality of household and living conditions in future health-related quality of life studies in the area.

\section{Acknowledgments}

The authors acknowledge all the GPs that participated in the study, as well as their patients.

\section{Funding}

This research received no specific grant from any funding agency in the public, commercial or not-for-profit sectors.

\section{Availability of data and materials}

No additional data are available.

\section{Authors' contributions}

FP and LS led the design of the study. FP performed the statistical analyses and drafted the manuscript. LS revised the manuscript critically for important intellectual content. Both authors read and approved the final manuscript draft.

\section{Competing interests}

The authors declare that they have no competing interests.

\section{Consent for publication}

Not applicable.

\section{Ethics approval and consent to participate}

The study was conducted in agreement with the principles of the Declaration of Helsinki. It was approved by the Ethics Committee of the Faculty of Health Sciences, University of Beira Interior, and the Ethics Committee of the Central Health Region of Portugal.

\section{Author details}

${ }^{1}$ Faculdade de Ciências da Saúde, Universidade da Beira Interior, Covilhã 6200-506, Portugal. ${ }^{2}$ Centro de Saúde de Aveiro, Aveiro 3810-000, Portugal.

${ }^{3}$ USF Topázio, Coimbra 3020-171, Portugal.

Received: 5 January 2016 Accepted: 4 November 2016

Published online: 11 November 2016

\section{References}

1. van den Akker M, Buntinx F, Knottnerus JA. Comorbidity or multimorbidity. Eur J Gen Pract. 1996;2:65-70.

2. Heyworth IT, Hazell ML, Linehan MF, Frank TL. How do common chronic conditions affect health-related quality of life? Br J Gen Pract. 2009;59:e353-358.

3. Goodman RA, Posner SF, Huang ES, Parekh AK, Koh HK. Defining and measuring chronic conditions: imperatives for research, policy, program, and practice. Prev Chronic Dis. 2013;10:E66.

4. Prazeres F, Santiago L. Prevalence of multimorbidity in the adult population attending primary care in Portugal: a cross-sectional study. BMJ Open. 2015;5:e009287.
5. Barnett K, Mercer SW, Norbury M, Watt G, Wyke S, Guthrie B. Epidemiology of multimorbidity and implications for health care, research, and medical education: a cross-sectional study. Lancet. 2012;380:37-43.

6. Brett T, Arnold-Reed DE, Troeung L, Bulsara MK, Williams A, Moorhead RG Multimorbidity in a marginalised, street-health Australian population: a retrospective cohort study. BMJ Open. 2014;4:e005461.

7. Habib RR, Mikati D, Hojeij S, El Asmar K, Chaaya M, Zurayk R. Associations between poor living conditions and multi-morbidity among Syrian migrant agricultural workers in Lebanon. Eur J Public Health. 2016;0:ckw096v1-ckw096.

8. Menotti A, Mulder I, Nissinen A, Giampaoli S, Feskens EJM, Kromhout D. Prevalence of morbidity and multimorbidity in elderly male populations and their impact on 10-year all-cause mortality. J Clin Epidemiol. 2001;54:680-6.

9. Smith SM, Soubhi H, Fortin M, Hudon C, O'Dowd T. Managing patients with multimorbidity: systematic review of interventions in primary care and community settings. BMJ. 2012;345:e5205.

10. Bayliss EA, Bayliss MS, Ware Jr JE, Steiner JF. Predicting declines in physical function in persons with multiple chronic medical conditions: what we can learn from the medical problem list. Health Qual Life Outcomes. 2004;2:47.

11. Alonso J, Ferrer M, Gandek B, Ware Jr JE, Aaronson NK, Mosconi P, Rasmussen NK, Bullinger M, Fukuhara S, Kaasa S, Leplège A. Health-related quality of life associated with chronic conditions in eight countries: Results from the International Quality of Life Assessment (IQOLA) Project. Qual Life Res. 2004;13:283-98.

12. Revicki DA. Health-related quality of life in the evaluation of medical therapy for chronic illness. J Fam Pract. 1989;29:377-80.

13. CDC. Measuring Healthy Days: Population Assessment of Health-related Quality of Life. Atlanta: GA: Centers for Disease Control and Prevention; 2000.

14. DeSalvo KB, Bloser N, Reynolds K, He J, Muntner P. Mortality prediction with a single general self-rated health question. A meta-analysis. J Gen Intern Med. 2006:21:267-75.

15. Michelson H, Bolund C, Brandberg Y. Multiple chronic health problems are negatively associated with health related quality of life (HRQoL) irrespective of age. Qual Life Res. 2000;9:1093-104.

16. Fortin M, Lapointe L, Hudon C, Vanasse A, Ntetu AL, Maltais D. Multimorbidity and quality of life in primary care: a systematic review. Health Qual Life Outcomes. 2004;2:51.

17. Fortin M, Bravo G, Hudon C, Lapointe L, Almirall J, Dubois MF, Vanasse A. Relationship between multimorbidity and health-related quality of life of patients in primary care. Qual Life Res. 2006;15:83-91.

18. McDaid O, Hanly MJ, Richardson K, Kee F, Kenny RA, Savva GM. The effect of multiple chronic conditions on self-rated health, disability and quality of life among the older populations of Northern Ireland and the Republic of Ireland: a comparison of two nationally representative cross-sectional surveys. BMJ Open. 2013;3:e002571.

19. Mujica-Mota RE, Roberts M, Abel G, Elliott M, Lyratzopoulos G, Roland M, Campbell J. Common patterns of morbidity and multi-morbidity and their impact on health-related quality of life: evidence from a national survey. Qual Life Res. 2015;24:909-18.

20. Salisbury C, Johnson L, Purdy S, Valderas JM, Montgomery AA. Epidemiology and impact of multimorbidity in primary care: a retrospective cohort study. Br J Gen Pract. 2011;61:e12-21.

21. Eurofound. Third European Quality of Life Survey - Quality of life in Europe: Subjective well-being. Luxembourg: Publications Office of the European Union; 2013.

22. Rosland AM, Heisler M, Piette JD. The impact of family behaviors and communication patterns on chronic illness outcomes: a systematic review. J Behav Med. 2012;35:221-39.

23. Magi M, Allander E. Towards a theory of perceived and medically defined need. Sociol Health IIIn. 1981;3:49-71.

24. Prazeres F, Santiago L. Multimorbidity in primary care in Portugal (MMPT): a cross-sectional three-phase observational study protocol. BMJ Open. 2014;4:e004113.

25. World Medical Association. World Medical Association Declaration of Helsinki: ethical principles for medical research involving human subjects. JAMA. 2013;310:2191-4.

26. von Elm E, Altman DG, Egger M, Pocock SJ, Gotzsche PC, Vandenbroucke $J P$, Initiative S. The Strengthening the Reporting of Observational Studies in Epidemiology (STROBE) statement: guidelines for reporting observational studies. J Clin Epidemiol. 2008;61:344-9.

27. Sousa L, Fiqueiredo D. (In)dependência na população idosa: um estudo exploratório na população portuguesa. Psychol. 2003;33:109-22. 
28. O'Halloran J, Miller GC, Britt H. Defining chronic conditions for primary care with ICPC-2. Fam Pract. 2004;21:381-6.

29. Kadam UT, Croft PR, North Staffordshire GPCG. Clinical multimorbidity and physical function in older adults: a record and health status linkage study in general practice. Fam Pract. 2007;24:412-9.

30. Pais-Ribeiro J. O importante é a saúde: estudo de adaptação de um instrumento para avaliar o estado de saúde. Lisboa: Fundação Merck Sharp \& Dohme; 2005

31. Ware Jr J, Kosinski M, Keller SD. A 12-Item Short-Form Health Survey: construction of scales and preliminary tests of reliability and validity. Med Care. 1996;34:220-33.

32. Larson CO. Use of the SF-12 Instrument for Measuring the Health of Homeless Persons. Health Serv Res. 2002;37:733-50.

33. Busija L, Pausenberger E, Haines TP, Haymes S, Buchbinder R, Osborne RH. Adult measures of general health and health-related quality of life: Medical Outcomes Study Short Form 36-Item (SF-36) and Short Form 12-Item (SF12) Health Surveys, Nottingham Health Profile (NHP), Sickness Impact Profile (SIP), Medical Outcomes Study Short Form 6D (SF-6D), Health Utilities Index Mark 3 (HUI3), Quality of Well-Being Scale (QWB), and Assessment of Quality of Life (AQoL). Arthritis Care Res (Hoboken). 2011;63 Suppl 11:S383-412.

34. Gandek B, Ware JE, Aaronson NK, Apolone G, Bjorner JB, Brazier JE, Bullinger M, Kaasa S, Leplege A, Prieto L, Sullivan M. Cross-validation of item selection and scoring for the SF-12 Health Survey in nine countries: results from the IQOLA Project. International Quality of Life Assessment. J Clin Epidemiol. 1998:51:1171-8.

35. Imperatori E. Ficha familiar dos centros de saúde: vantagens e dificuldades. Rev Port Sau Pub. 1985;3:51-6.

36. Agostinho M, Rebelo L. Família: do conceito aos meios de avaliação. Rev Port Clin Geral. 1988;32:6-17.

37. Smilkstein G. The family APGAR: a proposal for a family function test and its use by physicians. J Fam Pract. 1978;6:1231-9.

38. Smilkstein $G$, Ashworth $C$, Montano D. Validity and reliability of the family APGAR as a test of family function. J Fam Pract. 1982;15:303-11.

39. Dunn OJ. Multiple Comparisons Using Rank Sums. Technometrics. 1964;6: $241-52$.

40. Fortin M, Dubois MF, Hudon C, Soubhi H, Almirall J. Multimorbidity and quality of life: a closer look. Health Qual Life Outcomes. 2007;5:52.

41. Brettschneider C, Leicht H, Bickel H, Dahlhaus A, Fuchs A, Gensichen J, Maier W, Riedel-Heller S, Schafer I, Schon G, et al. Relative impact of multimorbid chronic conditions on health-related quality of life-results from the MultiCare Cohort Study. PLoS One. 2013;8:e66742.

42. Garin N, Olaya B, Moneta MV, Miret M, Lobo A, Ayuso-Mateos JL, Haro JM. Impact of multimorbidity on disability and quality of life in the Spanish older population. PLoS One. 2014;9:e111498.

43. Sprangers MAG, de Regt EB, Andries F, van Agt HME, Bijl RV, de Boer JB, Foets M, Hoeymans N, Jacobs AE, Kempen GIJM, et al. Which chronic conditions are associated with better or poorer quality of life? I Clin Epidemiol. 2000;53:895-907.

44. Wensing M, Vingerhoets $\mathrm{E}$, Grol R. Functional status, health problems, age and comorbidity in primary care patients. Qual Life Res. 2001;10:141-8.

45. Nutzel A, Dahlhaus A, Fuchs A, Gensichen J, Konig HH, Riedel-Heller S, Maier W, Schafer I, Schon G, Weyerer S, et al. Self-rated health in multimorbid older general practice patients: a cross-sectional study in Germany. BMC Fam Pract. 2014;15:1

46. Sprangers MAG, Schwartz CE. Integrating response shift into healthrelated quality of life research: a theoretical model. Soc Sci Med. 1999; 48:1507-15.

47. Kim Kl, Lee JH, Kim CH. Impaired health-related quality of life in elderly women is associated with multimorbidity: results from the Korean National Health and Nutrition Examination Survey. Gend Med. 2012;9:309-18.

48. Alonso-Moran E, Nuno-Solinis R, Orueta JF, Fernandez-Ruanova B, AldayJurado A, Gutierrez-Fraile E. Health-related quality of life and multimorbidity in community-dwelling telecare-assisted elders in the Basque Country. Eur 」 Intern Med. 2015;26:169-75.

49. Walker AE. Multiple chronic diseases and quality of life: patterns emerging from a large national sample, Australia. Chronic Illn. 2007;3:202-18.

50. Phillips $L$, Hammock RL, Blanton JM. Predictors of self-rated health status among Texas residents. Prev Chronic Dis. 2005;2:A12

51. Kaur H, Kaur H, Venkateashan M. Factors determining family support and quality of life of elderly population. Int J Med Sci Public Health. 2015:4 1049-53.
52. Chaves CB, Amaral OP, Nelas PA, Coutinho EC, Dionisio RM. Assessment of Family Functionality Among the Elderly With Chronic Illness. Eur J Counselling Psychol. 2013;2:139-44.

53. de Oliveira SC, Pavarini SC, Orlandi Fde S, de Mendiondo MS. Family functionality: a study of Brazilian institutionalized elderly individuals. Arch Gerontol Geriatr. 2014:58:170-6.

54. Wang MW, Huang YY. Evaluating family function in caring for a geriatric group: Family APGAR applied for older adults by home care nurses. Geriatr Gerontol Int. 2016;16:716-21.

55. Sok SR, Yun EK. A comparison of physical health status, self-esteem, family support and health-promoting behaviours between aged living alone and living with family in Korea. J Clin Nurs. 2011;20:1606-12.

56. de Araujo AA, Reboucas Barbosa RA, de Menezes MS, de II M, de Araujo Jr RF, de Medeiros CA. Quality of Life, Family Support, and Comorbidities in Institutionalized Elders With and Without Symptoms of Depression. Psychiatr Q. 2016;87:281-91.

57. Serra I, Gemito ML. Cuidadores informais: Quem quer ou quem pode? In: Mendes F, Gemito ML, Cruz D, Lopes M, editors. Enfermagem Contemporânea Dez temas, dez debates, vol. 1. Évora: Universidade de Évora; 2013. p. 133-49.

58. Griffith LS, Field BJ, Lustman PJ. Life stress and social support in diabetes: association with glycemic control. Int J Psychiatry Med. 1990;20:365-72.

59. Lett HS, Blumenthal JA, Babyak MA, Strauman TJ, Robins C, Sherwood A. Social support and coronary heart disease: epidemiologic evidence and implications for treatment. Psychosom Med. 2005;67:869-78.

60. Zautra AJ, Hoffman J, Potter P, Matt KS, Yocum D, Castro L. Examination of changes in interpersonal stress as a factor in disease exacerbations among women with rheumatoid arthritis. Ann Behav Med. 1997;19:279-86.

61. Rosland AM, Piette JD. Emerging models for mobilizing family support for chronic disease management: a structured review. Chronic Illn. 2010;6:7-21.

62. McRae I, Yen L, Jeon YH, Herath PM, Essue B. Multimorbidity is associated with higher out-of-pocket spending: a study of older Australians with multiple chronic conditions. Aust J Prim Health. 2013;19:144-9.

63. Lopes MJ, Mendes FRP, Martins SO, Ferreira PL. Acesso aos Cuidados de Saúde. Um direito em risco? Relatório Primavera 2015. OPSS/INODEs. 2015.

\section{Submit your next manuscript to BioMed Central and we will help you at every step:}

- We accept pre-submission inquiries

- Our selector tool helps you to find the most relevant journal

- We provide round the clock customer support

- Convenient online submission

- Thorough peer review

- Inclusion in PubMed and all major indexing services

- Maximum visibility for your research

Submit your manuscript at www.biomedcentral.com/submit
Biomed Central 